\title{
Daten, Fakten, Informationstechnologie ...
}

n der traditionellen Ausbildung der Mediziner spielt die Ökonomie der Gesundheit bisher keine große Rolle. Dies ändert sich schlagartig, wenn der Arzt im Krankenhaus oder in einer Praxis tätig wird. Je mehr Verantwortung der Arzt trägt, desto intensiver muss er sich mit den wirtschaftlichen Problemen befassen - dies gilt sowohl für die Praxis wie auch für die Klinik. Ein verantwortlicher Kliniker ist heutzutage im Prinzip täglich mit der Auseinandersetzung um die Finanzierung seiner Abteilung oder des gesamten Krankenhauses konfrontiert. Um adäquat reagieren zu können, muss er in der Lage sein, die Daten zu sichten und zu werten, die ihm die Verwaltung vorlegt.

Die Datenmenge hat in den letzten Jahren in einem kaum vorstellbaren Umfang zugenommen. In den 60er und 70er Jahren erhielt ein Chefarzt nur einmal im Monat eine Übersicht über die Belegung seiner Abteilung, die finanzielle Situation wurde in der Regel in größeren Abständen diskutiert. Details der Abrechnung - wie die Aufgliederung der einzelnen Einnahmen- und Ausgabenpositionen - wurden oft nur halbjährlich oder jährlich evaluiert.

Dies hat sich grundsätzlich gewandelt. Heute sind die Aufgaben eines Chefarztes ohne den Anschluss an das Intranet der Klinik nicht mehr zu bewältigen. Daten wie Belegung sind online praktisch jederzeit aktuell abzurufen. Die Verantwortlichen sind zeitnah in das Abrechnungssystem des Hauses eingeschaltet. Jeder leitende Arzt hat Zugriff zu den Krankenhausinformationssystemen (KIS) und sollte die Handhabung der Informationstechnologie beherrschen.

Ist die Hürde der Informationsbeschaffung genommen, steht die Wertung dieser Information an. Neben der Bewertung der klassischen Parameter, Erlöse und Ausgaben spielt hier für das Überleben der Abteilung die interne Leistungsverrechnung eine große Rolle. Ein entscheidender Punkt ist die Erfassung der aktuellen Leistung der eigenen Abteilung, um im internen Vergleich nicht zu kurz zu kommen. Die Beschäftigung mit diesen Daten setzt jedoch eine intensive Auseinandersetzung mit den Mechanismen und dem Ablauf der Prozeduren im Krankenhaus voraus, eine zeitaufwendig und oft frustrierende Aufgabe.
Entscheidend ist hier ein verlässliches Erfassungsund Abrechnungssystem innerhalb des Krankenhauses sowie eine gute und offene Kommunikation zwischen den Ärzten und der Verwaltung. Oft sind lange und zeitraubende Verhandlungen zwischen den leitenden Ärzten und Mitarbeitern der Verwaltung nicht zu umgehen, um das Zahlenwerk für alle transparent zu machen. Grundlage sind hierbei verlässliche Zahlen, welche die Verwaltung zur Verfügung stellen muss.

Die aktuelle Ausgabe des klinikarzt mit dem Themenschwerpunkt „Informatik in der Medizin“ präsentiert Ihnen Technologien und Systeme, die sie im Klinikalltag in Zukunft immer häufiger nutzen werden. Es ist zu erwarten, dass die administrativen Probleme in der Zukunft noch stärker in den Vordergrund drängen und so immer mehr die Aufmerksamkeit und Zeit der Ärzte in Anspruch nehmen. Und ohne diese modernen Techniken ist eine leitende Funktion im Krankenhaus schon heute kaum mehr denkbar.

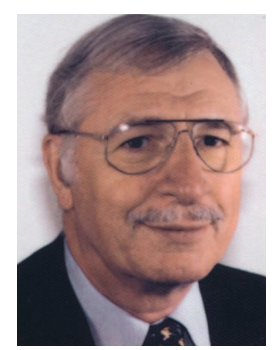

Prof. Dr. A. Weizel, Mannheim

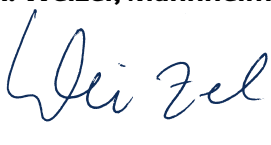

